\title{
Minimização da Poluição do Piloto em sistemas CDMA aplicando Algoritmo Genético
}

\author{
Sebastián Espinosa Rueda e Paulo Roberto Rosa Lopes Nunes
}

\begin{abstract}
Resumo- Nos sistemas CDMA, a existência de um grande número de sinais de pilotos com a mesma intensidade dentro de uma área geográfica (Poluição do Piloto) não é desejável porque degrada o desempenho do sistema. O objetivo deste trabalho é solucionar este problema minimizando a Poluição do Piloto dentro de uma região determinada, procurando, ao mesmo tempo, minimizar o número de usuários sem cobertura. O problema resultante é multi-objetivo, sugerindo a utilização de um Algoritmo Genético (AG). O AG utiliza as alturas das antenas das estações-base na representação do cromossoma. $O$ resultado consegue eliminar a maior parte da poluição, recomendando modificações no sistema para produzir resultados mais próximos ao ótimo.
\end{abstract}

Palavras-Chave- Algoritmos Genéticos, CDMA, Otimização, Problema Multi-Objetivo, Poluição do Piloto.

\begin{abstract}
In CDMA cellular systems the existence of a quite big number of pilot signals with the same signal strength inside a geographical area (Pilot Pollution) is not a desirable issue, because it causes degradation of system performance. The main objective of this article is to find a solution to this problem minimizing Pilot Pollution inside a delimited area, looking for the minimization of the number of users without any coverage, at the same time. The problem is multi-objective and suggests use of a Genetic Algorithm (GA) approach. The GA uses the height of antennas of the different base-stations in the chromosome representation. The results can eliminate most of the Pilot Pollution, suggesting some system modifications to reach the optimum.
\end{abstract}

Keywords - Genetic Algorithms, CDMA, Optimization, Multi-Objetive Problem, Pilot Pollution.

\section{INTRODUÇÃO}

O planejamento e otimização dos sistemas celulares baseados em tecnologia CDMA estão sustentados em simulações, dados estatísticos da rede e dados coletados em campo. A otimização do sistema é uma tarefa complexa e custosa, em função do tempo requerido, se for feita de maneira manual. Um dos maiores desafios é a minimização do fenômeno chamado Poluição do Piloto (Pilot Pollution) que é causador da degradação dos indicadores chave de desempenho do sistema tais como bloqueio e queda das ligações. Diferentemente das tecnologias D-AMPS e GSM, cuja otimização é baseada apenas em função da cobertura, em CDMA deve necessariamente ser considerado o tráfego

Sebastián Espinosa Rueda e Paulo Roberto Rosa Lopes Nunes, Seção de Engenharia Elétrica, Instituto Militar de Engenharia - IME, Praça General Tibúrcio 80, Praia Vermelha, 22290-270, Rio de Janeiro - RJ, Brasil. Emails: sebas_espinosa@yahoo.com, prnunes@ime.eb.br. gerado pelos usuários do sistema. Isto torna a otimização um processo Multi-Objetivo. Os Algoritmos Genéticos (AG) têm sido utilizados com bastante sucesso no tratamento da otimização multi-objetivo, fornecendo soluções automáticas, rápidas e eficientes. Os trabalhos disponíveis na literatura [1], [2], empregam os AGs principalmente no posicionamento das estações rádio-base (ERBs) para maximizar a cobertura dentro de um área específica. Nos últimos anos as empresas operadoras do serviço de telefonia celular têm instalado novas redes empregando as facilidades já existentes (co-siting). A abordagem feita neste trabalho está baseada em um co-siting, onde as coordenadas das ERBs são fixas, tendo como objetivo a minimização da Poluição do Piloto, maximizando a cobertura reversa (uplink) dos usuários dentro uma área geográfica com diferentes densidades de tráfego.

\section{A. Organização do Artigo}

O restante deste trabalho é organizado como apresentado a seguir. A descrição do problema, parâmetros do sistema e condições de teste são apresentados na Seção II. Na Seção III é apresentada a aplicação do algoritmo genético, representação do cromossoma e a descrição da avaliação e aptidão dos indivíduos. Na Seção IV estão os testes e resultados obtidos na resolução do problema. Finalmente, na Seção V, são apresentadas algumas conclusões e propostas de tarefas futuras.

\section{DESCRIÇÃO DO PROBLEMA}

\section{A. Poluição do Piloto em sistemas CDMA}

Dentro do processo de inicialização do terminal CDMA, uma estação móvel (EM) adquire o canal piloto geralmente da ERB mais próxima, i.e. aquela que tem melhor relação Ec/Io (Energia de Chip / Interferência) frente às outras ERBs, e que supera um determinado limiar. Os receptores Rake da EM permitem o processamento do sinal por até 3 pilotos, que pertencem à chamada lista de pilotos ativos [3]. Devido à convivência em co-siting, as ERBs podem originar a presença de um número não desejado de pilotos numa área específica, degradando a qualidade da comunicação.

A proposta apresentada neste trabalho é a otimização do sistema estabelecendo como objetivo maximizar a área de cobertura das ERBs minimizando as áreas onde existe poluição de piloto. Na literatura [4], [5], existem trabalhos onde diferentes variáveis são consideradas dentro da otimização, tais como tipo, azimuth e inclinação (tilt) das antenas; coordenadas das ERBs; potência do sinal piloto e até modificação dos parâmetros internos do padrão (lista de vizinhanças e parâmetros de soft-handoff, entre outros). Para 
simplificar a abordagem descrita neste artigo, as alturas das antenas de cada uma das ERBs foram considerados parâmetros variáveis, deixando os demais parâmetros do padrão constantes. Assim, o raio de cobertura das ERBs pode ser regulado com a altura da antena de cada ERB. Ao maximizar a área de cobertura, é preciso considerar a quantidade de usuários que podem estabelecer uma ligação com a estação rádio-base. O conceito de soft-capacity é introduzido para determinar a quantidade de tráfego em Erlangs que a ERB pode suportar sem bloquear o acesso aos usuários. Finalmente, define-se um mapa de tráfego, que corresponderá ao comportamento dos usuários dentro de unidades de área pré-definidas chamadas bins. Assim, o mapa poderá descrever as regiões onde existem mais usuários e, portanto onde existe maior demanda de tráfego.

\section{B. Raio de cobertura no Enlace Reverso (Uplink)}

Para determinar o raio de cobertura das estações rádiobase devem ser considerados vários parâmetros próprios de um sistema CDMA IS-95 que opera em uma área urbana garantindo cobertura em exteriores (outdoor). A perda de propagação foi caracterizada pelo modelo de Okumura-Hata com variação log-normal [6], [7]. Os parâmetros de funcionamento do sistema estão descritos nas Tabelas I e II

TABELA I

REQUISITOS DO SISTEMA

\begin{tabular}{|l|l|}
\hline Frequiência de Operação $[\mathrm{Mhz}]-f_{C}$ & 800 \\
\hline Largura de Banda $[\mathrm{Hz}]-W$ & 1228800 \\
\hline Taxa de Voz $[\mathrm{Kbps}]-R$ & 14,400 \\
\hline Ganho de Processamento $-G_{I 4,4}$ & 85,333 \\
\hline$E_{b} / I_{o}[\mathrm{~dB}]$ & 6 \\
\hline Fator de Atividade da Voz $-\alpha$ & 0,400 \\
\hline Ganho de Handoff [dB] $-G_{H O}$ & 4 \\
\hline Fator de Interferência Externa (Omni-Direcional) $\beta$ & 0,600 \\
\hline Porcentagem Cobertura Outdoor & 0,900 \\
\hline Fator de Carga ERB $-\mu$ & 0,600 \\
\hline Altura ERB [m] $-H_{E R B}$ (variável) & 30 \\
\hline Altura EM [m] $-H_{E M}$ & 1,500 \\
\hline Desvio padrão outdoor (margem cobertura) $[\mathrm{dB}]$ & 10 \\
\hline Constante De Propagação $-\gamma$ & 3,522 \\
\hline
\end{tabular}

O sistema oferece o serviço de voz com uma taxa de 14,4 Kbps na saída do vocoder (Rate Set 2). O Ganho de Processamento $\left(G_{14,4}\right)$ é a razão entre a Largura de Banda $(W)$ e a Taxa de $\operatorname{Voz}(R)$. O Fator de Atividade da Voz $(\alpha)$ representa a porcentagem de tempo que um usuário normalmente fala numa ligação telefônica, entre 40 e $50 \%$. A razão entre a Energia de Bit e a Densidade da Potência do Ruído $\left(E_{b} / I_{o}\right)$ é considerada por [3] como requisito de qualidade do enlace para atingir uma taxa de erro de quadro de $1 \%$. O Fator de Interferência Externa $(\beta)$ numa célula qualquer é a razão entre a interferência originada nas outras células do sistema e a interferência originada na mesma célula [7]. Para células omni o valor é de 0,6.

Assim, o número máximo de usuários $M_{M A X}$ por setor, é dado por:
$M_{M A X}=\left[\frac{G_{14,4}}{\alpha\left(E_{b} / I_{o}\right)} \frac{1}{(1+\beta)}\right]+1$.

TABELA II

PARÂMETROS DA ERB E EM

\begin{tabular}{|c|c|c|}
\hline & ERB & EM \\
\hline $\begin{array}{l}\text { Potência máxima transmissor }[\mathrm{dBm}] \\
-P_{T X E R B}(\text { na ERB), EIRP (na EM) }\end{array}$ & 36 & 28 \\
\hline Perda cabos $[\mathrm{dB}]-L_{E R B}$ & 2 & 0 \\
\hline Ganho antena $[\mathrm{dB}]-G_{A N T}$ & 10 & 0 \\
\hline Ganho diversidade $[\mathrm{dB}]-G_{D I V}$ & 3 & 0 \\
\hline Figura de ruído $[\mathrm{dB}]-F_{N}$ & 6 & 6 \\
\hline Porcentagem do piloto & $20 \%$ & $\begin{array}{l}\text { Não se } \\
\text { aplica }\end{array}$ \\
\hline Margem desvanecimento $-M_{F}$ & 7,700 & $\begin{array}{l}\text { Não se } \\
\text { aplica }\end{array}$ \\
\hline
\end{tabular}

O limiar da potência na recepção é dado por:

$$
\begin{aligned}
P_{R X \text { LIMIAR }}=-174 & +10 \log (W)+F_{N} \\
& -10 \log \left[\alpha(1+\beta)(1-\mu) M_{M A X}\right] .
\end{aligned}
$$

O nível mínimo de sinal necessário para a operação da EM, $R S L_{M I N}$ é dado por:

$R S L_{M I N}=P_{R X ~ L I M I A R}-G_{A N T}+L_{E R B}$.

A perda por propagação $\left(L_{O}\right)$, segundo o modelo de Okumura-Hata é:

$L_{O}=b+c \log \left(f_{C}\right)-13,83 \log \left(H_{E R B}\right)-3,2\left(\log 11,75 H_{E M}\right)^{2}$ $+4,97$

onde $b=69,55$ e $c=26,16$ para $f_{C}=800 \mathrm{MHz}$.

O nível de sinal $R S L_{M I N}$ também está em função do raio máximo de cobertura $r_{M A X}$ da célula, assim

$R S L_{M I N}=E I R P-L_{O}-10 \gamma \log r_{M A X}-M_{F}+G_{H O}+G_{D I V}$,

de onde temos:

$r_{M A X}=10^{\left[\frac{E I R P-R S L_{M I N}-L_{O}-M_{F}+G_{H O}+G_{D I V}}{10 \gamma}\right]}$.

\section{Capacidade da estação rádio-base (Soft Capacity)}

A carga oferecida pela estação base em Erlangs no enlace reverso $[\rho]$, deve considerar a probabilidade de bloqueio $P_{\text {bloqueio }}(2 \%)$ e pode ser determinada pelas seguintes equações [3]

$\rho=\frac{(W / R)(0,9)}{\left(E_{b} / I_{o}\right) \alpha(1+\mu)} f(B, \delta)$,

onde

$f(B, \delta)=\frac{1}{\delta}\left[1+\frac{\delta^{3} B}{2}\left(1-\sqrt{1+\frac{4}{\delta^{3} B}}\right)\right]$, 
sendo que

$$
\begin{aligned}
& B=\frac{\left(E_{b} / I_{o}\right)\left[Q^{-1}\left(P_{\text {bloqueio }}\right)\right]^{2}}{(W / R)(0,9)}, \mathrm{e} \\
& \delta=\exp \left(B^{2} \sigma^{2} / 2\right),
\end{aligned}
$$

onde $\sigma$ é o desvio padrão da potência no enlace reverso, igual a $2,5 \mathrm{~dB}$.

Assim, a soft-capacity da ERB ficou determinada em 21,32 Erlangs. Este valor é comparado com a quantidade de Erlangs gerada pelos usuários $\left(L_{T}\right)$ que têm cobertura da ERB. Se $L_{T}$ for maior que $\rho$ a ERB é penalizada por capacidade (ver Seção II-G).

\section{Determinação do Mapa de Tráfego}

O mapa de tráfego especifica o tráfego médio gerado pelos usuários em cada bin, e é determinado inicialmente empregando simulação Monte Carlo. A probabilidade de ter $n_{i}$ usuários no bin $i, \boldsymbol{P}\left(\boldsymbol{n}_{\boldsymbol{i}}\right)$, é dada pela distribuição de Poisson

$$
P\left(n_{i}\right)=\frac{a_{i}^{n_{i}}}{n_{i}} e^{-a_{i}},
$$

onde $a_{i}$ é o tráfego médio oferecido em cada bin. Conforme a distribuição de usuários experimental [usuários/bin] determinada no mapa de tráfego empregado, o tráfego gerado por usuário foi de $3 \mathrm{mE}$.

\section{E. Condições de teste}

Um sistema IS-95 foi simulado conforme as equações (1) a (11) para uma área de $50 \mathrm{~km}^{2}$ que representa parte de uma região urbana com aproximadamente 30 mil usuários divididos em 2500 bins com diferentes quantidades de usuários por bin, representando áreas com maior e menor demanda de tráfego. Foram consideradas 25 ERBs com alturas das antenas iniciais de 30 e 35 metros. Foi calculado o raio de cobertura de cada uma e a quantidade de usuários com cobertura de uma ou mais ERBs em cada bin. Os usuários cujos bins estão cobertos por mais de três ERBs têm poluição do piloto.

\section{F. Funções Custo}

As funções custo representam como os objetivos de otimização são avaliados e cumpridos. No presente trabalho foram definidas duas funções custo $C_{1}$ e $C_{2}$.

A função custo $C_{1}$ representa a porcentagem do tráfego total de todas as ERBs $T_{T O T}$, gerado por mais de 3 pilotos nos bins.

$$
C_{1}=\frac{\sum_{i=4}^{10} i T i}{T_{\text {TOT }}},
$$

onde $T_{i}$ é o tráfego total gerado no $\operatorname{bin} j$, servido por $i$ pilotos.

A função custo $C_{2}$ representa a porcentagem de $T_{T O T}$ dos bins que não possuem cobertura

$$
C_{2}=\left(1-\frac{T_{T O T}-T_{O}}{T_{T O T}}\right),
$$

onde $T_{O}$ é o trafego total dos usuários que não têm cobertura definido por

$T_{O}=\sum_{i=1}^{n} T i \beta i$,

onde $\beta i$ é o número de usuários no bin $i$.

\section{G. Penalização}

A função penalização é logarítmica e é calculada para a $\operatorname{ERB} j, 1 \leq j \leq 25$.

a) Penalização por Capacidade

$P_{1}=K_{1} \log \left(1+\frac{\Delta T}{K_{2}}\right)$,

onde $\Delta T=\left|L_{T j}-\rho\right|$.

Os valores de $K_{1}=150$ e $K_{2}=0,001$ foram obtidos experimentalmente. A carga de tráfego $L_{T j}$ é a quantidade total de Erlangs gerada pelos usuários com cobertura da $\mathrm{ERB} j$

\section{OTIMIZAÇÃo POR ALGORITMO GENÉTICO}

Os algoritmos genéticos (AG) são uma técnica de busca e otimização inspirada no principio Darwiniano de seleção natural e reprodução genética. São utilizados para encontrar soluções ótimas para funções de difícil modelagem matemática e problemas mal estruturados [8], [10]. A maioria dos AGs têm um ciclo na forma indicada na Figura 1.

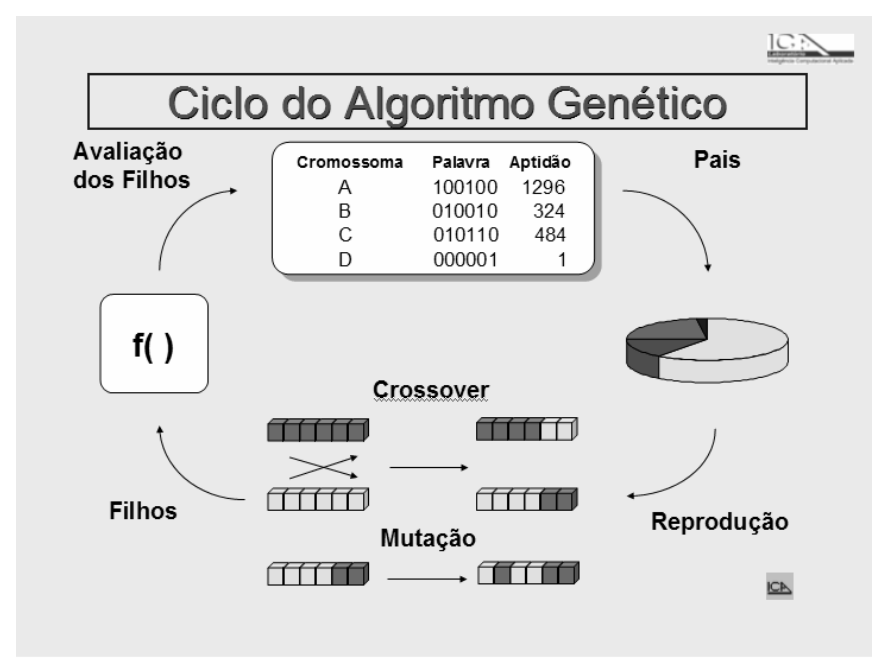

Fig.1. Ciclo do Algoritmo Genético

Cada indivíduo representa uma potencial solução ao problema. O AG aplica nos indivíduos operações de cruzamento e mutação, avaliando os novos indivíduos e selecionando de forma probabilística aqueles com maior avaliação para o seguinte ciclo (geração). O processo continua até satisfazer alguma condição de parada.

\section{A. Representação}

Neste trabalho o cromossoma representa as alturas das antenas das ERBs $\left(H_{E R B}\right)$ tendo, por tanto, 25 genes que contêm um número real cada um, entre $[10,30]$ ou $[25,45]$ 
metros, dependendo da localização da ERB. Considerando duas casas decimais, o número de bits necessários para representar um gene é de nove em cada caso. No total o cromossoma terá um comprimento de 225 bits, tornando o espaço de busca em $2^{225}$ possíveis soluções.

\section{B. Método utilizado / Operadores}

A ferramenta utilizada foi o software Evolver (www.palisade.com), já empregado na literatura [9]. O método usado foi o Recipe ou "Receita". Neste caso as alturas das antenas das ERBs poderão ter valores diferentes dentro dos intervalos especificados $[10,30]$ e $[25,45]$ metros.

Foram usados os operadores Cruzamento - Crossover e Mutação próprios do Evolver, variando os porcentagens de sorteio dos operadores.

\section{Avaliação}

A avaliação da aptidão do cromossoma é feita nas seguintes etapas:

1) Baseado nas considerações, valores dos parâmetros CDMA e equações (1) a (11) é calculado o raio $r_{E R B} j$ de cada ERB, para $1 \leq j \leq 15$.

2) As distâncias $d_{i j}$ entre os bins e cada ERB são calculadas. Se $d_{i j} \leq r_{E R B j}$

O bin $i$ tem cobertura da ERB $j, 1 \leq i \leq 2500$,

caso contrário (c.c)

$\mathrm{O}$ bin $i$ não tem cobertura da $\mathrm{ERB} j$.

3) Conta-se o número total de bins com cobertura de 1,2 e 3 ERBs; bins com cobertura de mais de 3 ERBs e bins sem cobertura.

4) Para cada ERB é determinada a carga de tráfego $L_{T}$ e Capacidade Reversa (Soft Capacity) $\rho$.

$$
\begin{aligned}
& \text { Se } L_{T}>\rho_{j}, \\
& \quad \text { Calcule } \Delta T_{j}=\left|L_{T j}-\rho_{j}\right|, \\
& \text { c.c. }
\end{aligned}
$$

$$
\Delta T_{j}=0 .
$$

5) O número de ERBs que oferecem cobertura para cada bin é determinada. O tráfego total coberto por todas as ERBs $\left(T_{T O T}\right)$ é calculado.

6) Determina-se o valor das funções custo $C_{1}$ e $C_{2}$, e a função penalização caso $L_{T}$ seja maior que $\rho$.

7) Agregação de Objetivos

Considerando as funções custo $C_{1}$ e $C_{2}$, os objetivos são minimizar as porcentagens do tráfego com poluição do piloto e de usuários sem cobertura. Assim, considerando uma soma ponderada deles temos que

$\min F=\min \left\{\left(\sum_{i=1}^{2} w_{i} C_{i}\right)+P_{1}\right\}$

onde $w_{i}$ representa o peso dado para cada objetivo e $P_{1}=\sum_{i=1}^{15} P_{1 i}$ é a penalização total por capacidade. O menor valor de $F$ gerado pelo cromossoma indicará uma melhor aptidão do indivíduo.

\section{RESUltados}

Foi desenvolvida uma simulação do mapa da poluição do piloto representando os bins servidos por um ou mais pilotos e aqueles sem cobertura, baseado nos requisitos do sistema. As Figuras 2 e 3 apresentam os mapas de poluição do piloto antes e depois da otimização.

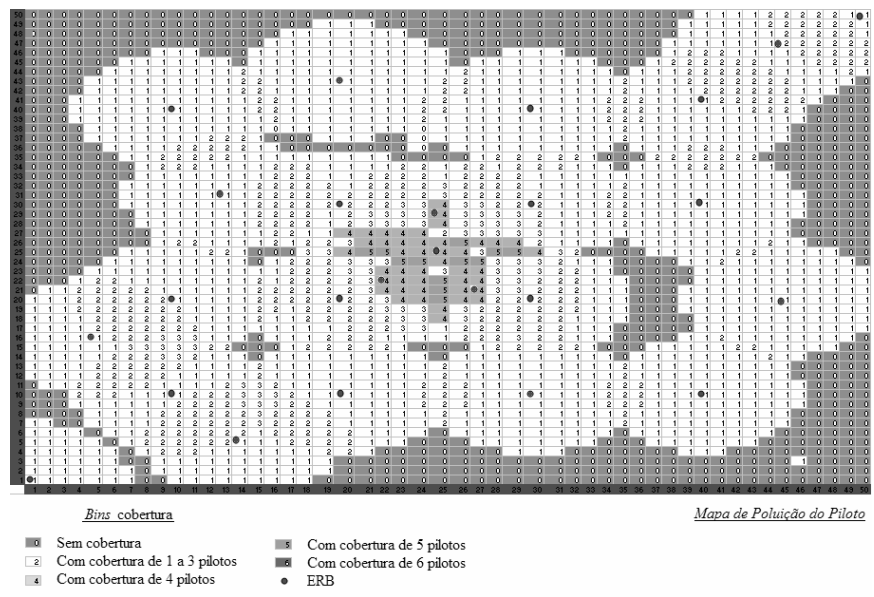

Fig. 2. Mapa de Poluição do Piloto antes da otimização.

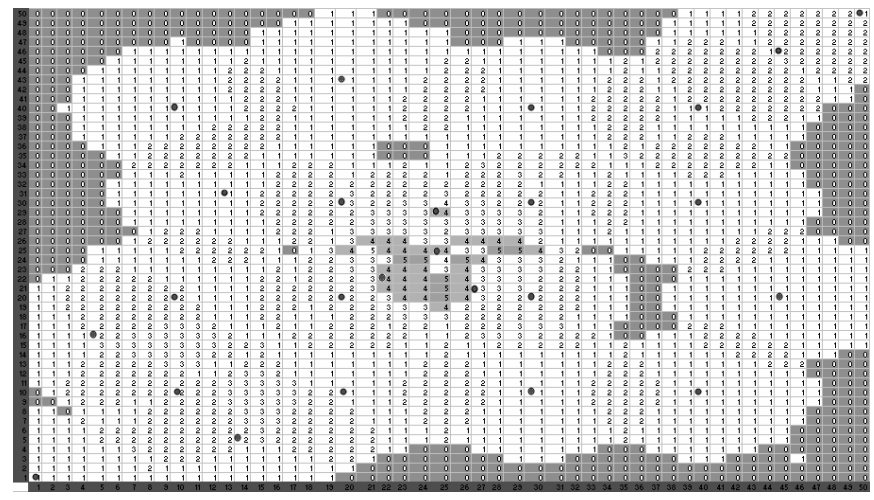

Fig. 3. Mapa de Poluição do Piloto depois da otimização.

A diminuição da poluição do piloto é evidente na região central da área geográfica. As áreas sem cobertura não aumentaram. $\mathrm{O}$ AG variou as $H_{E R B}$ das ERBs tratando de evitar a penalização. As variáveis de desempenho avaliadas na otimização são apresentadas na Tabela III.

TABELA III

DESEMPENHO DO AG

\begin{tabular}{|l|c|c|}
\hline & Inicio Otimização & Final Otimização \\
\hline Custo $1-C_{l}$ & 0,218120 & 0,155682 \\
\hline Custo $2-C_{2}$ & 0,481017 & 0,133632 \\
\hline $\begin{array}{l}\text { Custo Total - Penalty } \\
{\left[C_{T}-P\right]}\end{array}$ & 5,028293 & 1,492006 \\
\hline $\begin{array}{l}\text { F.Penalty por Capacidade } \\
\left(P_{l}\right)\end{array}$ & 0,000000 & 0,000000 \\
\hline $\begin{array}{l}\text { Usuários sem cobertura } \\
\text { (USC) }\end{array}$ & 2022 & 1079 \\
\hline Custo Total + Penalty $\left[C_{T}+P\right]$ & 5,028293 & 1,492006 \\
\hline
\end{tabular}

Foram consideradas 2000 iterações e os valores de pesos $w_{1}=1$ e $w_{2}=10$. O valor da probabilidade de Crossover $\left(P_{C}\right)$ foi 0,60 e da Mutação $\left(P_{M}\right)$ foi de 0,10 e a População 
Inicial foi 150 . Os resultados obtidos indicam que o AG cumpre o objetivo proposto embora achando que o co-siting não é suficiente para diminuir a quantidade de usuários sem cobertura. Será necessário um investimento adicional na rede para instalar novas ERBs nas áreas sem cobertura. Vários experimentos foram executados para observar o efeito da variação dos valores dos pesos $w$, população inicial e operadores genéticos. Os resultados são apresentados na Tabela IV e indicam que:

$\mathrm{O}$ peso $w_{2}$ deve ser maior que $w_{1}$ para poder minimizar o número de usuários sem cobertura.

Os valores padrão dos operadores genéticos atingem os melhores valores das funções $C_{1}$ e $C_{2}$, e $\left(C_{T}-P\right)$.

A capacidade máxima da ERB não é atingida, por tanto nenhum experimento tem penalização.

Aumentar a população não diminui o número de usuários sem cobertura nem consegue o menor $\left(C_{T}+P\right)$.

Alterar os valores das probabilidades do crossover e mutação, também não influi na minimização dos usuários sem cobertura.

TABELA IV

EXPERIMENTOS

\begin{tabular}{|l|c|c|c|c|c|}
\hline $\begin{array}{l}\text { Valores } \\
\text { finais }\end{array}$ & $\begin{array}{c}w_{I}=10 \\
w_{2}=1\end{array}$ & $\begin{array}{c}\text { Pop_size } \\
=75\end{array}$ & $\begin{array}{c}\text { Pop_size }= \\
300\end{array}$ & $\begin{array}{c}P_{C}=0,80 \\
P_{M}=0,05\end{array}$ & $\begin{array}{c}P_{C}=0,30 \\
P_{M}=0,30\end{array}$ \\
\hline$C_{l}$ & 0,000000 & 0,156162 & 0,191434 & 0,189373 & 0,191923 \\
\hline$C_{2}$ & 0,244435 & 0,134800 & 0,131693 & 0,130331 & 0,135471 \\
\hline$C_{T}-P$ & 0,244435 & 1,504166 & 1,508362 & 1,492679 & 1,546636 \\
\hline$P_{1}$ & 0,000000 & 0,000000 & 0,000000 & 0,000000 & 0,000000 \\
\hline USC & 1304 & 1076 & 1076 & 1079 & 1112 \\
\hline$C_{T}+P$ & 0,244435 & 1,504166 & 1,508362 & 1,492679 & 1,546635 \\
\hline
\end{tabular}

\section{CONCLUSÕES}

O Algoritmo Genético da ferramenta Evolver, configurado para enfrentar o problema descrito neste trabalho, conseguiu os objetivos projetados: minimizar o número de pilotos servidores em cada bin e minimizar o número de bins -usuários- sem cobertura. Foram encontrados os valores mais adequados dos parâmetros do AG para desenvolver a solução do problema. Embora o mínimo ideal $F=0$, não tenha sido atingido, a função penalização conseguiu manter os valores de capacidade dentro de intervalos válidos para as ERBs. O estudo pode-se estender para regiões maiores ou com mais densidade de usuários ou ERBs, modificando os parâmetros do AG como população inicial o valores dos pesos $w_{i}$ para obter os resultados desejados. Como tarefa futura é sugerido considerar a implementação de um ambiente de simulação completo do sistema CDMA (IS-95, 1xRTT ou WCDMA) que considere, entre outros, controle de potência no enlace direto e reverso, ambientes de soft e softer-handoff, parâmetros de controle do CDMA, informação precisa do terreno, predições de cobertura e demais variáveis do sistema (antenas diretivas, setorização, lista de vizinhanças, azimuth e tilt das antenas), de modo que os resultados possam ser avaliados no sistema simulado retornando as avaliações ao AG (feedback) para continuar seu processo evolutivo.

\section{REFERÊNCIAS BIBLIOGRÁFICAS}

[1] L. Raisanen e R. Whitaker, Comparison and Evaluation of Multiple Objetive Genetic Algorithms for the Antenna Placement Problem. Mobile Networks and Applications 10, Springer Science, 2005.

[2] G. Cabral, K. Lage, G. Mateus e R. Franqueira, Planejamento de Redes Celulares de Terceira Geração considerando localização de Estações Radio Base, Controle de Potência e Múltiplos Serviços. Departamento de Ciência da Computação, UFMG, 2004.

[3] S. Yang, CDMA RF System Engineering. Artech House Publishers, c. 4, 7, 8 e 10, 1998.

[4] R. Menolascino, M. Pizarroso, C. Lepschy e B. Salas, Third Generation Mobile Systems Planning Issues. VTC'98, IEEE, 1998.

[5] S.B. Jamaa, Z. Altman, J. Picard, B. Fourestié e J. Mourlon, Manual and Automatic Design for UMTS Networks. Mobile Networks and Applications 9, Kluwer Academic Publishers, 2004.

[6] K. Kim, Handbook of CDMA System Design Engineering and Optimization. Prentice-Hall, c. 9 e 10, 2000.

[7] L. Silva-Mello, Planejamento de Sistemas Celulares $e$ Radio Acesso - Apostilas e Notas de Aula. CETUC, PUC-RIO, 2006.

[8] M. Pacheco, "Algoritmos Genéticos: Princípios e Aplicações” e Computação Evolucionária - Apostila e Notas de Aula. ICA, PUC-RIO, 2006.

[9] R. Bezerra, M. Pacheco, M. Velasco e M. Grivet, Optimization of Frequency Assignment for a Cellular Mobile System by Genetic Algorithms. Departamento de Engenharia Elétrica - ICA - CETUC, PUC-RIO, 2001

[10] D. Goldberg, Genetic Algorithms in Search, Optimization and Machine Learning. Addison-Wesley Publishing Company, 1989. 